\title{
Evaluación de derechos de naturaleza individual, social y colectivos en el ámbito de la crianza familiar ${ }^{1}$
}

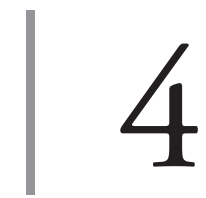

\author{
https://doi.org/10.21830/9789585284845.04
}

Manuel Bermúdez-Tapia ${ }^{2}$
Universidad Privada San Juan Bautista

\section{Introducción}

En una evaluación jurídica, los casos más complejos son observados en el ámbito de las relaciones familiares, contexto que se agudiza si el conflicto familiar se judicializa, principalmente, porque se generan circunstancias de orden jurídico, social e incluso familiar aún más complejas.

Surge en tal sentido, una evaluación de derechos, intereses y contextos que implica el análisis de derechos de naturaleza individual, social, familiar, colectivos y otras áreas difusas, especialmente, porque el ámbito de las relaciones familiares —en lo íntimo, lo privado y lo público — es sumamente especial, por lo cual en el derecho de familia se aceptan elementos probatorios, procesales y sustantivos diferentes a los que usualmente se ejecutan en otras disciplinas jurídicas.

Como de referencia sustancial, puede observarse el hecho de que un divorcio llegue a provocar una indemnización a manera de compensación, situación que, en el ámbito de la evaluación de la responsabilidad de las partes en conflicto, suele estudiarse en el contexto del "daño". Este punto de referencia permite identificar por qué en el contexto familiar es posible no asimilar valores sustantivos y procesales típicos de contextos normativos liberales, en

1 Este capítulo se deriva del proyecto de investigación Análisis de la Institucionalidad Democrática en el Perú, desarrollado en la Facultad de Derecho de la Universidad Privada San Juan Bautista.

2 Abogado de la Pontificia Universidad Católica del Perú (magna cum laude). Magíster en Derecho. Doctorado en Derecho por la Pontificia Universidad Católica de Argentina. Profesor-investigador de la Universidad Privada San Juan Bautista y profesor de la Facultad de Derecho de la Universidad Nacional Mayor de San Marcos. Orcid: http://orcid.org/0000-0003-1576-9464 - Contacto: manuel.bermudez@upsjb. edu.pe 
los cuales la presencia (y exigencia) de un ámbito tuitivo se hace sumamente referencial e incluso excluyente.

Por ello, la mejor ocasión para evaluar contextos en los que hay complementación, contraposición, yuxtaposición y contradicción entre tipos de derechos se da en el ámbito familiar, especialmente, cuando se examinan casos que implican la crianza de los hijos. Ante este panorama, se presenta un caso de evaluación de la jurisprudencia peruana, tanto a nivel constitucional (Tribunal

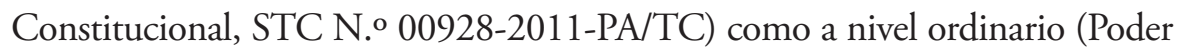
Judicial, jurisprudencia sobre alimentos, tenencia, divorcio y violencia familiar).

\section{Límites y parámetros objetivos en la crianza familiar}

Por los alcances de la jurisprudencia peruana, en particular respecto de la capacidad de los progenitores para ejecutar las actividades cotidianas de crianza, consideramos oportuno someter los alcances de estas facultades a una evaluación crítica, puesto que estos temas revisados cotidianamente en el ámbito jurisdiccional ordinario constituyen la más alta tasa de referencialidad de casos en el Poder Judicial peruano. De acuerdo con este parámetro, se trabajan los apartados siguientes.

\subsection{Facultades y límites de los derechos paternales frente a la crianza de los hijos}

En la bibliografía especializada sobre la temática, existe información referencial sobre las obligaciones de los progenitores y un severo vacío de información sobre los derechos de estos sobre sus hijos. Por esta razón, planteamos de forma preliminar la necesidad de establecer límites, tanto en forma positiva como negativa (Schaffer, 2000, p. 266). Los límites positivos se materializan en la defensa de los eventuales derechos de los hijos, mientras que los negativos están vinculados a los perjuicios producidos en el hijo cuando, a nombre de este, se ejecutan determinados actos.

Complementariamente, existe otro elemento catalizador de evaluaciones: las condiciones de los progenitores. Los progenitores en situación matrimonial 
o convivencial ejercen de forma conjunta la patria potestad, con lo cual los conflictos sobre la determinación de derechos en la crianza de los hijos no suelen suceder o llegan a determinarse luego de superar un debate familiar interno. En el caso contrario, cuando los progenitores están separados o son divorciados, la práctica permite señalar que existe una situación que limita los derechos del progenitor en cuanto su derecho a la tenencia, situación principalmente provocada por el otro progenitor, sin importar si el perjudicado provocase o no la crisis familiar.

\subsection{El contexto punitivo y sus límites}

En términos comparativos, es posible mencionar el artículo 262 del Código Civil colombiano, en el que se regula en términos generales la facultad de imponer sanciones a los hijos como facultad de los progenitores, lo cual forma parte del ejercicio de la patria potestad. Sin embargo, dicha facultad tiene como límite natural y constitucional el uso de la fuerza en la imposición de sanciones a los hijos, tanto en una modalidad física como emocional.

Según este límite, la Corte Constitucional colombiana legitima el derecho a corregir a los hijos, siempre y cuando la sanción sea razonable respecto de las condiciones del menor. Así, los límites para establecer su razonabilidad están detallados en el artículo 262 del Código Civil colombiano, que son los siguientes :
a. La sanción debe ser sobre actos ciertos y probados
b. La sanción debe tener una utilidad educativa
c. La sanción debe ser proporcional a la falta cometida
d. La sanción debe imponerse en forma oportuna
e. La sanción no puede contener elementos de violencia física sobre el menor

\subsection{El derecho a corregir durante la crianza}

Por los alcances mismos de este punto, se debe considerar que en la actualidad la mayoría de legislaciones en el contexto comparado pondera que el progenitor a cargo de la patria potestad ejerza el deber de orientar a su hijo, a efectos de encaminarlo en las prácticas sociales cotidianas. 
Dado el carácter psicológico del proceso de formación de todo menor de edad, los progenitores se ven limitados en su facultad de imponer una sanción, por ello resulta importante analizar el alcance del deber de corregir en la crianza para así generar en el hijo a una mejor formación (Herrera \& Spaventa, 2007). De forma excepcional y ante una situación extrema, el progenitor puede imponer una sanción, pero esta debe ser moderada, a efectos de lograr una comprensión en el hijo del error cometido.

\subsection{La evaluación de los derechos de cada progenitor con respecto del otro progenitor}

La equivalencia de derechos y condiciones en las obligaciones es absoluta entre los progenitores (Ramírez, 2011), sin embargo, esto se puede modificar en función de la separación o divorcio de las partes, en los casos en los que hubo un matrimonio. Por tal motivo, el ejercicio de la patria potestad será el elemento referencial en el que se determinen los derechos de cada uno de los progenitores y también las condiciones con las cuales estos derechos se establecerán con respecto de los otros derechos del progenitor que no cuenta con la tenencia o el contacto inmediato de su hijo o progenie.

\section{I.4.I RESPECTO DE LA RELACIÓN CON EL OTRO PROGENITOR}

Dentro de las características generales de los procesos de crisis familiar, los progenitores deben tener en cuenta que las causas que provocaron dicha situación no pueden afectar los derechos de la contraparte en cuanto a su relación con los hijos. Esta situación no suele ser tomada en cuenta en los contextos en los que el conflicto se judicializa, por lo tanto, conviene tener presente que surgen tres contextos totalmente autónomos:

i. Los derechos del primer progenitor

ii. Los derechos del segundo progenitor

iii. Los derechos de los hijos respecto de cada uno de sus progenitores (Urra \& Urra, 2015, p. 76)

En este punto corresponde detallar que, a raíz del caso Reynaldo Shols (STC N. ${ }^{\circ}$ 09332-2006-PA/TC) en complementación de la STC N. ${ }^{\circ} 01317-$ 2008-HC/TC, caso Hermanos Tudela van Breugel-Douglas, un cónyuge de 
una pareja con hijos no podría imponer una limitación a los derechos de esta a relacionarse con sus hijos, conforme se establece una familia ensamblada generada por una separación o divorcio preexistente, sobre la cual se genera una evolución de la comprensión de "la familia" con base en el vínculo familiar (Bermúdez-Tapia, 2011).

En función de lo detallado, cada progenitor está condicionado a establecer mecanismos de relación, crianza y vínculo con su familia (Acuña San Martín, 2015, p. 101), lo cual puede exponerse de la siguiente manera:

i. No limitar, condicionar (económicamente) o variar el régimen de visitas en forma arbitraria.

ii. No puede provocar situaciones de alienación parental, de padrectomía u obstrucción de vínculo entre el hijo con el otro progenitor. (Bolańos, 2008, p. 35)

La referencia de este punto proviene de los alcances de la Casación N.o 2067-2010 Lima, de acuerdo con la cual la Corte Suprema evaluó la incidencia del síndrome de alienación parental:

i. No provocar un alejamiento entre el hijo y la pareja sentimental tras la finalización de la relación. (Bermúdez-Tapia, 2012, p. 410 )

ii. No provocar casos de obstrucción en la relación con los abuelos u otros miembros familiares, en función a lo que Quinn (2002) detalla como una situación inaceptable en el ámbito de la evaluación de derechos en el contexto contemporáneo. (Bermúdez-Tapia, 2011, p. 411)

\section{I.4.2 RESPECTO DEL EJERCICIO DE PATRIA POTESTAD Y LA TENENCIA EXCLUSIVA}

Por el contexto subjetivo que suele vincularse al contexto familiar en crisis, es conveniente detallar que el progenitor asignado con la tenencia de los hijos, desarrolla estos elementos:

Ejercicio directo del derecho. Quien tiene asignado este derecho por vía judicial debe ejercerlo de modo directo, sin alguna consideración que pudiera modificar la ratio decidendi del juez. Esto limita o condiciona negativamente el hecho de que el progenitor con la tenencia a su favor traslade el cuidado de sus hijos a terceras personas (abuelos o familiares). Condición que no limita ni 
genera una situación negativa si el progenitor con dicho derecho, al ejecutar sus actividades ordinarias, no dispone de un tiempo exclusivo para criar a sus hijos. Esto por tanto corresponderá a la evaluación práctica de los casos.

Ejercicio de la tenencia. Se debe tener en cuenta que el progenitor no puede realizar actividades que incidan arbitrariamente en el desarrollo del hijo. Los hijos, al ser sujetos de derechos, no pueden quedar supeditados a la anulación de estos, porque uno de los progenitores le imponga una condición que no responde a un elemento objetivo y razonable.

\section{Los derechos y el contexto de la crianza de hijos}

Surge una serie de elementos en evaluación en el contexto contemporáneo que merecen ser evaluados en forma autónoma a continuación.

\subsection{Elementos provenientes de la atención del derecho a la salud}

Los hijos, al estar al cuidado de sus progenitores, pueden verse involucrados en situaciones en las que se deba evaluar su salud, sin embargo, esto está determinado en función a algunos límites, como los siguientes:

a. Los tratamientos médicos deben ser autorizados por especialistas en la salud. Las condiciones de crecimiento de los hijos suelen estar relacionadas con factores que afectan su salud: todo tratamiento clínico, quirúrgico o médico (en general) debe estar relacionado con alguna indicación de un especialista. Esto evitaría la especulación y la ejecución de actos que pudieran afectar la integridad física del hijo o su propia salud.

b. Las intervenciones quirúrgicas deben ser autorizadas por los dos progenitores. Tomando en cuenta la situación de incapacidad que tienen los hijos menores de edad para decidir de manera autónoma respecto de ciertos riesgos, es necesario que los dos progenitores autoricen la intervención quirúrgica que se requiera. Esto permite detallar que toda intervención que no sea necesaria resulta limitante a toda facultad de un progenitor para ejecutar alguna acción 
quirúrgica, así sea estética, por cuanto podría provocar una condición negativa tanto en lo físico como en lo psicológico en una persona en desarrollo.

c. Respecto de la alimentación en referencia a la salud de los hijos. Elemento muy especial y contemporáneo, porque permite detallar las situaciones de obesidad, bulimia u otro desorden alimenticio, los cuales constituyen problemas en el ámbito de la salud pública y pueden configurar una condición que implica maltrato infantil (Mardomingo, 2003)

\subsection{Evaluación de la salud psicológica}

Muy a pesar de que los progenitores suelen ponderar la defensa de sus hijos en el contexto del conflicto familiar judicializado, estos no toman en cuenta algunos problemas que inciden en el ámbito psicológico en la formación de sus propios hijos. En tal sentido, los progenitores no son conscientes de situaciones como estas:

a. La generación de actos de alienación parental. Los condicionamientos negativos de un progenitor (generalmente el que queda con la tenencia luego de una decisión judicial) sobre el otro constituyen un elemento que tiene repercusiones psicológicas en los hijos, pues estos últimos, hacia la edad madura, llegan a generar contextos de violencia en sus propias familias (Bermúdez-Tapia, 2012).

b. La generación de situaciones de obstrucción de vínculo. Estas situaciones hacen que los hijos se sientan en medio del conflicto y se vean afectados por las condiciones de este, gracias a sus propios progenitores (Estévez et al., 2007).

c. El contexto de padrectomía. Cuando la figura de uno de los progenitores no logra ser permanente en el desarrollo de los hijos, estos asumen una condición negativa respecto del progenitor ausente que se amplifica en su edad adulta, lo cual provoca un rechazo absoluto de patrones conductuales, que suelen extenderse de forma independiente respecto de su propia familia. 


\subsection{Evaluación de los derechos vinculados al ámbito de la intimidad y la salud sexual}

Este es un punto absolutamente crítico en la crianza de los hijos, en particular, porque la autonomía personal de estos no está totalmente bajo el control de los progenitores. No obstante, la jurisprudencia permite detallar algunas referencias, como las que se enumeran a continuación:

a. Interrupción de embarazos en adolescentes. De acuerdo con los análisis de la legislación peruana, los menores de edad están condicionados por un factor social en cuanto a la gestión pública de la salud reproductiva:

i. Los menores de edad tienen un acceso reducido o limitado a mecanismos de control de natalidad,

ii. Los menores de edad tienen una limitada (casi nula) capacidad para optar por la continuidad de un embarazo no deseado, como ocurre por ejemplo en España, donde la Interrupción Voluntaria del Embarazo está aprobada legislativamente, a través de la Ley Orgánica 2/2010 de salud sexual y reproductiva. (Begońa y Mendiola, 2010, p. 320)

En Colombia, es posible traer a lugar una sentencia relacionada con este tema. La Corte Constitucional facultó a una niña de 12 años y a su madre para llevar a cabo la interrupción del embarazo, con el argumento de que la menor tenía problemas de frustración y depresión, lo que provocaría complicaciones obstétricas. El contenido de dichos elementos proviene del Expediente T-841/11 (Corte Constitucional de Colombia, 2011), acción de tutela instaurada por un progenitor, en representación de su hija menor de edad, en contra de BB. E.P.S., jurisprudencia referencial para el ámbito de evaluación.

b. La ablación como práctica cultural reprimida en paises occidentales. En el contexto comparado, existen procesos judiciales iniciados en países europeos en los que se ha sancionado a los padres, incluso con la condena privativa de libertad, por realizar procedimientos de ablación en sus hijas menores de edad (Toubia \& Rahman, 2000). 
Los progenitores, sobre todo aquellos provenientes de países africanos o asiáticos, en defensa de las prácticas de ablación, sustentan sus derechos de vincular a sus hijas en prácticas culturales nativas que, a su juicio, resultan constitucionales, aún en países en los cuales tienen condición de migrantes. La jurisprudencia europea no admite dichos argumentos y sanciona con penas severas semejantes prácticas, sin importar que sus practicantes sean médicos o chamanes.

Las medidas limitativas de derechos vinculados al pluralismo jurídico de inmigrantes en Europa se extiende incluso a la manifestación de valores religiosos en público. En este sentido, Francia limita la facultad de los padres de vincular a la práctica religiosa a sus hijos, prohibiendo que estos exhiban sus valores religiosos en sitios públicos (De Haro, 2011). Esta prohibición alcanza los usos del burka, el nicab, el hiyab y los crucifijos. Dicha iniciativa, actualmente es seguida en Italia, Holanda y Dinamarca.

La autonomía de los progenitores para inducir a los hijos en prácticas culturales, sociales y religiosas — como la exhibición de atuendos y símbolos religiosos-, sobre todo en Francia, está limitada a la valoración de la dignidad del menor de edad, principalmente porque este menor, en su interacción social con otros, puede sufrir procesos de discriminación, exclusión y marginación. Esta limitación opera en sitios públicos, pero no se extiende a los ámbitos privados de la familia, en los que los progenitores asumen total disposición del menor en una práctica cultural y sociorreligiosa.

c. Circuncisión a varones menores de edad. En Estados Unidos de América, existe una extensa bibliografía de casos jurisprudenciales sobre la circuncisión en menores de edad. Se citan casos vinculados al análisis de la capacidad para efectuar dicha práctica quirúrgica, para diferenciarlos de la mayor parte de la jurisprudencia, que está vinculada a la mala praxis en la ejecución de la intervención quirúrgica:

i. Caso: Kalina vs. General Hospital of the City of Syracuse (100 NYS2d 226, 18 A. D. 2d 757 (1962). La Corte de Nueva York determinó que la circuncisión era un asalto a 
la integridad del menor, porque este no tiene la capacidad suficiente para actuar conforme a sus derechos.

ii. Caso: Union Pacific Railway Company vs. Botsford, 141 U.S. 250 (1891), y

iii. Caso: Planned Parenthood vs. Casey, 505 U. S. 833 (1992).

En los dos últimos casos, la Corte Suprema de Justicia determinó que la práctica de la circuncisión solo elimina tejido saludable que no afecta la funcionalidad del órgano sexual masculino, con lo cual determinó que su ejecución afectaba la salud, sin embargo, decretó que se trata de una violación a la integridad corporal, en función de la determinación de dicha práctica en un menor de edad.

En Queensland (Australia), se debatió legislativamente la facultad de los progenitores para ejecutar la práctica de la circuncisión en sus hijos menores de edad y se llegó a la determinar que esta es ilegal, que no respeta los derechos de los niños a decidir sobre su condición clínica. Este debate surgió por el desarrollo jurisdiccional de casos vinculados a la práctica de la circuncisión (Queensland Law Reform Commission, 1993).

En Holanda, a raíz de la eliminación de toda forma de práctica de la ablación, la Asociación Médica Holandesa (1993) planteó la prohibición de la práctica de la circuncisión, porque los sometidos quirúrgicamente son demasiado jóvenes para dar su consentimiento para la intervención.

En Argentina, se ha registrado la variación de la tenencia a favor de la madre cuando el padre ejecutó el procedimiento de circuncisión al hijo de ambos, sin autorización. El padre, alegando derechos vinculados al ámbito religioso (era judío), ejecutó la práctica a pesar de la oposición de la madre católica. La justicia argentina determinó que los progenitores, al tener plenos derechos y ejercer conjuntamente la patria potestad del menor, debían concordar en practicar la circuncisión y que, ante el desacuerdo mostrado, se le otorgaba la tenencia a la madre, ante la separación de hecho de ambos (Fernández, 2017, p. 238). 


\subsection{En el ámbito de la educación}

Otro de los problemas propios de la crianza de los hijos se evidencia en el ámbito educativo. Dichos problemas se podrían solucionar con la correcta aplicación de la tenencia compartida, pero la autodeterminación del progenitor con tenencia suele ser el patrón característico. El progenitor con la tenencia suele excluir la participación del otro en la educación del hijo, en particular, en el seguimiento académico, aun en la misma educación superior. La limitación en el acceso al seguimiento escolar para el progenitor sin tenencia constituye una característica que los mismos colegios asumen a favor del progenitor con tenencia, a efectos de que este no retire al hijo del centro escolar (y no los perjudique económicamente).

La situación se agrava si el progenitor con tenencia ejecuta una variación del centro educativo del hijo, sin consentimiento o comunicación con el otro. Los progenitores sin tenencia en el Perú, para efectos de no provocar un mayor conflicto con el otro progenitor o ante la eventualidad de que sus denuncias no tengan eco en el sistema judicial, autolimitan sus derechos, sin tomar en cuenta el carácter autónomo de estos.

En España, en cambios ante este tipo de situaciones, se admite el derecho del progenitor sin tenencia (generalmente el padre) a acceder al registro académico y hacer un seguimiento académico de su hijo, sin limitación, porque este hace uso de su ejercicio de la patria potestad, la cual se diferencia del régimen de tenencia.

\section{Conclusiones}

El contexto familiar permite examinar una serie de situaciones en las que cada integrante de la familia en crisis puede evaluar el ámbito de sus propios derechos y obligaciones en un contexto independiente y autónomo de los intereses, derechos y obligaciones respecto de sus propios familiares.

Las referencias a la autonomía personal, respecto de la jurisprudencia evaluada, permiten detallar que la individualidad de la persona debe también revisarse respecto de las relaciones familiares, en las que, en ciertas situaciones, 
los derechos deben ser evaluados de forma independiente. En este sentido, la evaluación de la casuística requiere la empleabilidad de principios y reglas que procuren ponderar unos derechos sobre la proyección de los derechos de otra persona en función de un elemento superior por su ámbito tuitivo.

Surge, por tanto, la necesaria evaluación de los alcances de los derechos de la persona en una proyección a los derechos de naturaleza individual, colectiva, familiar o, eventualmente, los que estén vinculados a una proyección de intereses difusos, donde la ponderación y la evaluación de una controversia deben estar focalizadas en la atención de lo razonable de una medida, en particular, cuando surgen contradicciones a nivel intergeneracional.

De este modo, la concepción de la condición de sujeto de derecho debe entenderse en un ámbito amplio, pero guardando correlación con el contexto en evaluación, por cuanto los derechos de los hijos se ven inmersos en la proyección de los derechos de los progenitores, quienes a su vez tienen una obligación sobre ellos, que es la de protegerlos y velar por su integridad.

\section{Referencias}

Acuña San Martín, M. (2015). Derecho de relación entre los hijos y el progenitor no custodio tras el divorcio. Dykinson.

Begoña, A., \& Mendiola, M. (2010). Cuestiones en torno a la Ley Orgánica 2/2010, de 3 de marzo, de Salud Sexual y Reproductiva y de la Interrupción Voluntaria del Embarazo. En F. Bandrés Moya, \& S. Delgado Bueno, Biomedicina y derecho sanitario. Además Comunicación.

Bermúdez-Tapia, M. (2011). Redefiniendo el derecho de familia en la tutela del vínculo familiar en la jurisprudencia peruana. Revista de Derechos Fundamentales, 5, 43-62.

Bermúdez-Tapia, M. (2012). Derecho procesal de familia. Aproximación critica no convencional a los procesos de Familia. Editorial San Marcos.

Bolaños, I. (2008). Hijos alienados y padres alienados: mediación familiar en rupturas conflictivas. Reus.

Corte Constitucional de Colombia. (2011). Sentencia T-841/11. http://www.corteconstitut cional.gov.co/relatoria/2011/T-841-11.htm

De Haro, F. (2011). Un mundo en transición. Encuentro.

Estévez, E., Jiménez, T., \& Musitu, G. (2007). Relaciones entre padres e hijos adolescentes. Nau Libres.

Fernández Espinoza, W. (2017). La alienación parental como causa de variación de la tenencia. Vox Iuris, 33(1), 223-240. 
Herrera, M., \& Spaventa, V. (2007). Vigilar y castigar: el poder de corrección de los padres. En R. Bergalli, \& I. Rivera Bieras (Eds.), Desafios, jóvenes y adultos. El difícil vínculo social (pp. 94-124). Antrophos.

Mardomingo, M. (2003). Psiquiatría para padres y educadores: ciencia y arte. Narcea.

Queensland Law Reform Commission. (1993). Circumcision of male infants [Research paper]. http://www.cirp.org/library/legal/QLRC/

Quinn, G. (2002). Derechos Humanos y Discapacidad. Naciones Unidas.

Ramírez, D. (2011). Crianza compartida, en equidad. Un derecho humano. Editorial Acad.

Schaffer, R. (2000). Desarrollo social. Siglo XXI.

Toubia, N., \& Rahman, A. (2002). Female genital mutilation: a practical guide to worldwise laws and practices. Zed.

Urra, J., \& Urra, B. (2015). Padres en conflicto con sus hijos. Participación Educativa, 4(7), 75-80. 